The Effect of Zufa Versus Chlorhexidine Gluconate Mouthwashes on

\title{
Oral Flora of Patients Under Mechanical Ventilation in the Intensive Care Unit: A Double-Blind, Randomized Clinical Trial
}

Abbasali Ebrahimian (iD ${ }^{1}$, Raheb Ghorbani (iD ${ }^{2}$, Mehrooz Alishah (iD ${ }^{3}$, Alieh Zamani-Kiasari ${ }^{4}$, Niloofar Kianvash-Rad (iD ${ }^{5}$ and Monir Nobahar (iD ${ }^{1,2,6, *}$

${ }^{1}$ Nursing Care Research Center, Semnan University of Medical Sciences, Semnan, Iran

${ }^{2}$ Social Determinants of Health Research Center, Semnan University of Medical Sciences, Semnan, Iran

${ }^{3}$ Student Research Committee, Nursing and Midwifery School, Semnan University of Medical Sciences, Semnan, Iran

${ }^{4}$ Department of Anesthesiology and Critical Care, Mazandaran University of Medical Sciences, Mazandaran, Iran

${ }^{5}$ Department of Cosmetic and Restorative, Dental School, Semnan University of Medical Sciences, Semnan, Iran

${ }^{6}$ Medical-Surgical Nursing Group, Nursing and Midwifery School, Semnan University of Medical Sciences, Semnan, Iran

"Corresponding author: Medical-Surgical Nursing Group, Nursing and Midwifery School, Semnan University of Medical Sciences, Semnan, Iran. Email: nobahar43@semums.ac.ir

Received 2019 January 20; Revised 2019 May 02; Accepted 2019 May 07.

\begin{abstract}
Background: Aromatic herbs and spices contain essential oils and are recognized by their considerable antimicrobial activity. One of the natural mouthwashes in traditional Iranian medicine is Zufa. It is not clear, however, that the extract of Zufa can affect oral health as much as Chlorhexidine gluconate, which is a potent antimicrobial mouthwash.

Objectives: This study aimed to compare the effect of Zufa and Chlorhexidine gluconate mouthwashes on oral flora of patients under mechanical ventilation in ICUs.

Methods: This double-blind, randomized clinical trial was conducted on the ninety-ICU-patients receiving mechanical ventilation in a university-affiliated hospital in Sari, Iran, from June 2017 to March 2018. The patients were randomly divided into three groups ( $\mathrm{n}=30$ ) using block randomization method; the Zufa, Chlorhexidine gluconate, and normal saline groups; the patients' mouth were washed with $15 \mathrm{~mL}$ of the Zufa $0.02 \%$, Chlorhexidine gluconate $2 \%$, or normal saline $0.09 \%$, 30 seconds, twice a day (eight am and four pm), for 3 days, respectively. Oral hygiene status was evaluated before the intervention, and then up to three days after that by the Beck oral assessment scales (BOAS).

Results: The data of BOAS showed no significant difference between the Zufa, Chlorhexidine gluconate, and normal saline groups before the intervention phase ( $\mathrm{P}>0.05$ ), and in this phase BOAS means in the Zufa, Chlorhexidine gluconate, and normal saline were $6.0 \pm 40.56,6.0 \pm 43.72$, and $6.47 \pm 0.62$, respectively. A significant association was found between the BOAS score after mouthwashes and the oral health of the patients in the three groups $(\mathrm{P}>0.05)$.

Conclusions: Based on the results of our study, mouthwashes of Zufa and normal saline showed the same effectiveness as Chlorhexidine gluconate on the oral health of intubated patients hospitalized in the ICU.
\end{abstract}

Keywords: Chlorhexidine Gluconate, Health, Hygiene, Intensive Care Units, Iran, Mechanical Ventilation, Mouthwashes, Oral, Traditional Medicine, Zufa

\section{Background}

In healthy individuals, oral flora remains constant over time. However, after admission to the hospital, the oral flora changes into the gram-negative microorganisms that have more pathogenicity $(1,2)$. Microbes that are naturally present in the mouth are harmless Saprophyte (Alphahemolytic Streptococcus, Lactobacillus), which cause no invasive infections. However, they might cause infections in critically ill patients in intensive care units (ICUs)(3). These infections are more prevalent in intubated patients due to the creation of a direct path for the entrance of various bacteria into the mouth (4). Therefore, prevention of the occurrence of microbial colonization in hospitalized patients by proper performing of oral health instructions is one of the priorities of nursing care (5). Oral health in intubated patients usually includes different activities such as oral cavity examination, oral cavity cleansing with an oral swab or tooth brushing, suctioning, mouthwash, and mouth moisturizing $(1,6)$. 
Chlorhexidine gluconate is a cationic biguanide biocide, first introduced in 1940 by the British imperial chemical industries (ICI) (7). This mouthwash has an inhibitory effect on gram-negative bacteria, gram-positive bacteria, and yeasts (8). However, it is associated with complications, including change of sense of taste, dryness, and burning of the mouth, change in the color of the teeth and adverse systemic effects if swallowed (9). The positive efficiency of chlorhexidine in oral health has been studied extensively. Kandwal et al. in A randomized controlled clinical trial study showed that the chlorhexidine gel could be effective in reducing plaques and gingival index (10). Hua et al. showed that chlorhexidine mouthwash reduced the risk of ventilator-associated pneumonia (VAP) in critically ill patients receiving mechanical ventilation from $24 \%$ to $18 \%$ (11). Some recent studies have incited controversy about chlorhexidine mouthwashes. Deschepper et al. in observational cohort study showed that chlorhexidine mouthwash $(\leq 300 \mathrm{mg}$ ) was associated with increased risk of death in patients (12). In another study, Price et al. found chlorhexidine mouthwash to be associated with an increased risk of mortality in general ICUs (13). Today, nurses attempt to improve productivity in patient care through various measures, including the use of medicinal plants.

Aromatic herbs and spices contain essential oils and are recognized by their considerable antimicrobial activity $(14,15)$. Herbal mouthwashes exert more efficiency compared to those containing chemical compounds, due to their natural ingredients in terms of their compatibility with body physiology and lower poisoning probabilities $(16,17)$. One of the natural mouthwashes in traditional Iranian medicine is Zufa (18) or Hyssopus Angustifolius L, which is a shrub of the peppermint family (Lamiaceae) and is used as herbal medicine in traditional medicine (19). This perennial plant has small line leaves and blue-purple flowers (20). The height of its bush is $20-25 \mathrm{~cm}$, and the length and width of its leaves are two-four $\mathrm{cm}$ and 0.5 $1 \mathrm{~cm}$, respectively (21). The antibacterial, antifungal, and antioxidant features of this plant are due to the presence of pinocamphone, isopinocamphone, and beta-Pinene, respectively (22). Antimicrobial tests have shown that hyssopus affects Staphylococcus aureus and Candida albicans (23). It is not clear whether the extract of Zufa can affect oral health as much as Chlorhexidine gluconate, which is a potent antimicrobial mouthwash. Therefore, with regard to the importance of oral health in intubated patients and the benefits of herbal combinations and their lower level of complications in comparison to chemical compounds, this study was conducted.

\section{Objectives}

The study aimed to compare the effect of Zufa and Chlorhexidine gluconate mouthwashes on oral flora of patients under mechanical ventilation in ICU.

\section{Methods}

\subsection{Study Design and Settings}

This study was a double-blind, randomized clinical trial (ID: IRCT2017060711399N5). Patients were unconsciousness, and did not know the type of mouthwash. The mouthwash was prepared by the main researcher, and the person performing the oral health procedure was unaware of the type of the mouthwash. This study was performed from June 2017 to March 2018.

\subsection{Sample}

The research sample consisted of all ICU-hospitalized patients receiving mechanical ventilation in the Imam Khomeini Hospital in Sari, Iran. This hospital is the general hospital in Mazandaran province in the north of Iran. This governmental hospital is referral, with 11 sections and 382 beds. The inclusion criteria were GCS below eight, being intubated, and under mechanical ventilation, lack of allergy to plants of the peppermint family (according to evidencebased statements of the families of the patients), natural teeth, no diabetes, having a gastrointestinal tube and the lack of receiving food from mouth, no damage to the oral cavity, no consumption of immunosuppressive drugs, and no seizures. The exclusion criteria were patient discharge from the ICU or death of the patients before the completion of the intervention, lack of cooperation of the legal guardians or companions of the patients, allergy to the applied mouthwashes, and incubation before the completion of the intervention.

\subsection{Measures}

Data collection tool was comprised of a questionnaire related to demographic characteristics, which assessed age, gender, occupational status, level of education, and underlying diseases; in addition, Beck oral assessment scale (BOAS), which contains five subscales (lips, mucosa and gingiva, tongue, teeth, and saliva). In this scale, each subscale is scored within the range of one-four based on the oral health of patients. The minimum and maximum scores are 5 and 20, respectively, where lower scores are indicative of lack of problems, and higher scores demonstrate more problems in this regard. In general, the score of five shows lacks of impairment, whereas the scores of $6-10,11-15$, and 16 - 20 are indicative of mild, moderate, 
and severe impairments, respectively. It is notable that the reliability and validity of the BOAS were confirmed in Safarabadi and Ghaznavirad research (24).

\subsection{Samples}

The sample size was estimated after performing preliminary research on 18 patients who met the inclusion criteria. In this preliminary study, the improvement of oral health problems four days after receiving mechanical ventilation was calculated at $18.2 \%$ and $92.8 \%$ in the Zufa and Chlorhexidine gluconate groups, respectively. Then 10 patients were entered each group (30 in total) with the confidence of $99 \%$ and power of $95 \%$. Considering subject dropout, a total of 90 patients were enrolled in the research. Equation 1 was used to calculate the sample size.

$n=\frac{2\left(Z_{1-\frac{\alpha}{2}}+Z_{1-\beta}\right)^{2} P(1-P)}{\left(P_{1}-P_{2}\right)^{2}}$

\subsection{Materials}

In the current research, the applied solutions were normal saline $(0.9 \%$ sodium chloride, made by Iranian Parenteral and Pharmaceutical Co.), Chlorhexidine gluconate 2\% (made by Behsa Pharmaceutical Co., Arak, Iran), and 0.02\% Zufa (prepared by the Natural Resources and Agriculture Research and Education Center in Mazandaran, Iran).

\subsection{Ethical Considerations}

Before collecting the data, the study protocol was approved by the Ethics Committee of Semnan University of Medical Sciences (No.: IR.SEMUMS.REC.1396.5), followed by explaining the objectives of the research to the patients and obtaining written informed consent.

\subsection{Procedures}

The participants were randomly divided into three groups of 30 subjects (A, B, C), including A (Zufa), B (Chlorhexidine gluconate), and $C$ (normal saline). For random allocation of the patients, A, B, and C cards (30 of each) were coded with numbers 1-30 and put into envelopes. The first patient entering the research received one of the cards and was allocated to a group based on the written code. This process continued until reaching 30 patients. Before the intervention, the oral health of the patients was evaluated using BOAS. After recording the scores, the researcher provided two syringes containing five $\mathrm{mL}$ of mouthwash solution for the nurse based on the type of the group. It is worth noting that the nurse had received the necessary education regarding a standard mouth rinse.
To initiate the process, the trained nurse rinsed all parts of the mouths of patients (tongue, mucous and gums, teeth and soft and hard palates). Then the mouths of the patients were cleaned with soft baby toothbrushes, placing the toothbrush at a 45-degree angle to the gum line to penetrate underneath it. The internal and external surfaces of the upper and lower teeth were cleaned by brushing from the gum to the crown. To clean the abrasive surface of the teeth, the toothbrush hair was placed parallel to the tooth surface and slowly moved forward and backward. The sides of the teeth were cleaned by moving the toothbrush back and forth. After finishing the brushing, different parts of the mouths were rinsed with the second syringe. In the following, the remaining oral solution in the mouths was suctioned in less than 30 seconds using a white nelaton catheter.

The mouthwash program was continued twice a day (eight am and eight pm) every day for up to three days. In addition, BOAS was applied to check the oral health status of the patients for up to three days after the intervention (at eight am and before the mouthwash).

\subsection{Data Analysis}

Data analysis was performed with SPSS Statistics for Windows, version 16.0 (SPSS Inc., Chicago, Ill, USA), using descriptive statistics (mean \pm standard deviation (SD)), Chi-square, Fisher's exact test, and independent $t$-test. Since the results of the Shapiro-Wilk test was indicative of lack of normal distribution of the data, the Kruskal-Wallis and Friedman's tests were applied for independent threelevel and multilevel variables, respectively. In addition, a significance level of 0.05 was considered statistically significant.

\section{Results}

The present study was conducted between May 16th 2017 and March 29th 2018, in which 513 individuals were admitted to the ICU of Imam Khomeini Hospital of Sari. In total, 393 patients failed to meet the inclusion criteria, and 32 patients excluded from the research (Figure 1). Finally, 88 individuals remained, including 56 (63.64\%) males and $32(36.36 \%)$ females. The minimum and maximum ages of the patients were 18 and 78 years, respectively. All of the patients were homogeneous in terms of age, gender, occupational status, and type of disease $(\mathrm{P}>0.05)$ (Table 1$)$. However, there were differences between the three study groups regarding the level of education $(\mathrm{P}=0.008)$. Before the intervention, $98.9 \%$ of the patients had mild oral health problems (Table 2).

In addition, BOAS means in the Zufa, Chlorhexidine gluconate, and Normal saline were 6.0 \pm 40.56, 6.0 \pm 43.72 


\begin{tabular}{|c|c|c|c|c|}
\hline \multirow[t]{2}{*}{ Variables } & \multicolumn{3}{|c|}{ Frequency in Groups } & \multirow[t]{2}{*}{ PValue } \\
\hline & Zufa & Chlorhexidine & Normal Saline & \\
\hline Age, $y$ & $49.33 \pm 17.86$ & $57.00 \pm 19.34$ & $50.72 \pm 18.12$ & $0.18^{\mathrm{b}}$ \\
\hline \multicolumn{5}{|l|}{ Gender } \\
\hline Male & $20(66.7)$ & $19(63.3)$ & $18(60)$ & $0.86^{\mathrm{c}}$ \\
\hline Female & $10(33.3)$ & $11(36.7)$ & $12(40)$ & \\
\hline Type of job & & & & $0.24^{\mathrm{c}}$ \\
\hline Government's employee & $1(3.3)$ & $2(6.7)$ & $4(13.3)$ & \\
\hline Proletarian & $2(6.7)$ & $0(0)$ & $2(6.7)$ & \\
\hline Unemployed & $11(36.7)$ & $18(60)$ & $15(50)$ & \\
\hline Retired & $8(26.7)$ & $7(23.3)$ & $3(10)$ & \\
\hline Others job & $8(26.7)$ & $3(10)$ & $6(20)$ & \\
\hline Cause of hospitalization & & & & $0.36^{\mathrm{c}}$ \\
\hline Cardiovascular disease & $8(26.7)$ & $10(33.3)$ & $10(33.3)$ & \\
\hline Gastrointestinal diseases & $1(3.3)$ & $5(16.7)$ & $4(13.3)$ & \\
\hline kidney diseases & $1(3.3)$ & $0(0)$ & $0(0)$ & \\
\hline Respiratory diseases & $3(10)$ & $3(10)$ & $4(13.3)$ & \\
\hline Surgical problems & $5(16.7)$ & $1(3.3)$ & $2(6.7)$ & \\
\hline Trauma & $12(40)$ & $11(36.7)$ & $10(33.3)$ & \\
\hline The habit of smoking & & & & $0.18^{\mathrm{c}}$ \\
\hline Yes & $10(33.3)$ & $10(33.3)$ & $8(26.7)$ & \\
\hline No & $20(66.7)$ & $20(66.7)$ & $22(73.3)$ & \\
\hline
\end{tabular}

Abbreviation: SD, standard deviation.

${ }^{\mathrm{a}}$ Values are expressed as No. (\%) or mean \pm SD.

${ }^{\mathrm{b}}$ Kruskal Wallis test.

${ }^{\mathrm{c}}$ Chi-square test.

and $6.47 \pm 0.62$, respectively. According to the results, no significant difference was observed in BOAS means of the groups before the intervention $(\mathrm{P}>0.05)$. Moreover, all three groups were homogeneous regarding oral health status before the intervention (Table 3 ).

After the intervention, the oral health status of $66.7 \%$ of the subjects in the Zufa group significantly improved. Similar results were observed in $70 \%$ and $60 \%$ of the participants in the Chlorhexidine gluconate and normal saline groups, respectively. On the second day of the intervention process, the levels of the improvement in the oral health of subjects of the Zufa, Chlorhexidine gluconate and normal saline groups were reported to be $90 \%$, 93.3\%, and $76.7 \%$, respectively.

On the third day of the intervention, there was $96.7 \%$, $93.3 \%$, and $80 \%$ improvement in the oral health of the subjects in the Zufa, Chlorhexidine gluconate, and normal saline groups, respectively (Table 2 ). In addition, the results of the Kruskal-Wallis test were indicative of the sig- nificant difference between the effectiveness of the mouthwashes used for improving the oral health of patients in the three groups $(\mathrm{P}>0.05)$ (Table 3). Furthermore, the Friedman's test demonstrated that the applied mouthwashes had positive impacts on the oral health status of the patients under mechanical ventilation in the three study groups (Table 4).

\section{Discussion}

In the present study, the oral health status of the evaluated patients was unfavorable upon admission to ICU and before the intervention. In addition, $98.9 \%$ of the patients had mild oral health problems, and no significant difference was observed between the study groups in terms of BOAS score before the intervention. In the research conducted by Safarabadi and Ghaznavirad the effect of Echinacea mouthwash and Chlorhexidine gluconate on the oral health of intubated patients in ICU was evaluated. In 


\begin{tabular}{|c|c|c|c|c|}
\hline \multirow[t]{2}{*}{ BOAS/Score } & \multicolumn{3}{|c|}{ Groups } & \multirow[t]{2}{*}{ Total Groups } \\
\hline & Zufa & Chlorhexidine & Normal Saline & \\
\hline \multicolumn{5}{|c|}{ Before the intervention } \\
\hline 5 & $0(0)$ & $1(3.5)$ & $0(0)$ & $1(1.1)$ \\
\hline $6-10$ & $29(100)$ & $28(96.5)$ & $30(100)$ & $87(98.9)$ \\
\hline $11-15$ & $0(0)$ & $0(0)$ & $0(0)$ & $0(0)$ \\
\hline $16-20$ & $0(0)$ & $0(0)$ & $0(0)$ & $0(0)$ \\
\hline \multicolumn{5}{|c|}{ The day after the intervention } \\
\hline 5 & $20(69)$ & $21(72.4)$ & $18(60)$ & $59(67.0)$ \\
\hline $6-10$ & $9(31)$ & $8(27.6)$ & $12(40)$ & $29(33.0)$ \\
\hline $11-15$ & $0(0)$ & $0(0)$ & $0(0)$ & $0(0)$ \\
\hline $16-20$ & $0(0)$ & $0(0)$ & $0(0)$ & $0(0)$ \\
\hline \multicolumn{5}{|c|}{ Two days after the intervention } \\
\hline 5 & $26(89.6)$ & $27(93.1)$ & $23(76.7)$ & $76(86.4)$ \\
\hline $6-10$ & $3(10.34)$ & $2(6.9)$ & $7(23.3)$ & $12(13.6)$ \\
\hline $11-15$ & $0(0)$ & $0(0)$ & $0(0)$ & $0(0)$ \\
\hline $16-20$ & $0(0)$ & $0(0)$ & $0(0)$ & $0(0)$ \\
\hline \multicolumn{5}{|c|}{ Three days after the intervention } \\
\hline 5 & $28(96.5)$ & $28(96.5)$ & $24(80)$ & $80(90.9)$ \\
\hline $6-10$ & $1(3.5)$ & $1(3.5)$ & $6(20)$ & $8(9.1)$ \\
\hline $11-15$ & $0(0)$ & $0(0)$ & $0(0)$ & $0(0)$ \\
\hline $16-20$ & $0(0)$ & $0(0)$ & $0(0)$ & $0(0)$ \\
\hline
\end{tabular}

Abbreviation: BOAS, Beck oral assessment scales.

${ }^{\mathrm{a}}$ Values are expressed as No. (\%).

\begin{tabular}{|c|c|c|c|c|}
\hline \multirow[t]{2}{*}{ Times } & \multicolumn{3}{|c|}{ BOAS Mean Scores } & \multirow[t]{2}{*}{ PValue } \\
\hline & Zufa & Chlorhexidine & Normal Saline & \\
\hline Before the intervention & $6.40 \pm 0.56$ & $6.43 \pm 0.72$ & $6.47 \pm 0.62$ & 0.934 \\
\hline The day after the intervention & $5.33 \pm 0.47$ & $5.33 \pm 0.54$ & $5.47 \pm 0.62$ & 0.653 \\
\hline Two days after the intervention & $5.10 \pm 0.30$ & $5.07 \pm 0.25$ & $5.23 \pm 0.43$ & 0.136 \\
\hline Three days after the intervention & $5.03 \pm 0.18$ & $5.07 \pm 0.25$ & $5.20 \pm 0.40$ & 0.077 \\
\hline
\end{tabular}

Abbreviation: BOAS, Beck oral assessment scales.

${ }^{\mathrm{a}}$ Values are expressed as mean $\pm \mathrm{SD}$

the mentioned research, $80 \%$ of the patients had moderate oral health problems, and results of the intervention showed no significant difference between the intervention and control groups regarding oral health (24).

Baradari et al. compared the impacts of herbal and Chlorhexidine gluconate $2 \%$ mouthwashes on patients receiving mechanical ventilation. According to their results, all of the intubated patients in the ICU had Staphylococcus aureus and Pneumococcus before the mouthwash pro- cess (25). This shows that the majority of patients admitted to the ICU had some levels of oral health problems and required advanced oral care immediately after admission. According to the results of the current research, a significant difference was observed between the BOAS score on the fourth day of intervention and BOAS scores before the intervention in all three groups. In addition, all three types of mouthwash (normal saline, Zufa, and chlorhexidine gluconate) improved the oral health status of intu- 


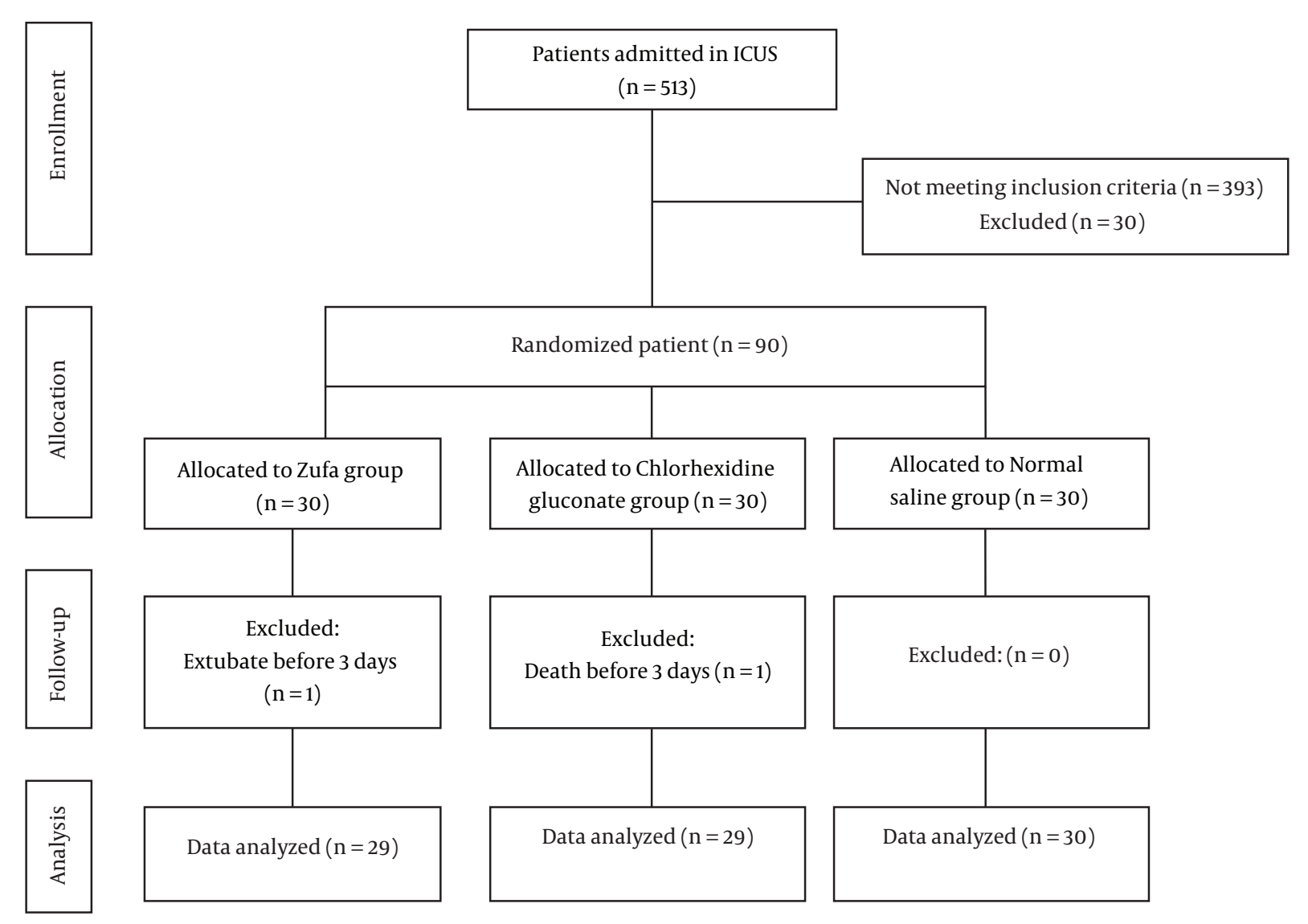

Figure 1. The flow of the patients participated in the trial

bated patients under ventilation. Different studies have evaluated the effectiveness of various mouthwashes compared to Chlorhexidine gluconate mouthwash.

In some of the previous studies, the evaluated mouthwashes have shown equal effectiveness as Chlorhexidine gluconate. In a study by Vangipuram et al. on the effectiveness of aloe vera and Chlorhexidine gluconate mouthwashes on periodontal health of medical students, aloe vera had the same effectiveness as Chlorhexidine gluconate (26). However, some other mouthwashes were not as effective as Chlorhexidine gluconate in some other studies. In Baradari et al. study, herbal mouthwashes showed a weaker anti-bacterial effect in the oral environment compared to chlorhexidine gluconate (25). Researchers have not reported the equal efficacy of an herbal mouthwash as Chlorhexidine gluconate so far.

\subsection{Conclusions}

In the present research, the mouthwashes of Zufa and normal saline had the same effectiveness as Chlorhexidine gluconate on the oral health of intubated patients hospitalized in ICU. The similarity between the mouthwashes was due to the oral health status (mild problems) of the patients upon admission to the ward. Therefore, all three types of mouthwash seem to improve the mild oral problems. Moreover, timely and proper oral care along with the use of the three types of mouthwash was able to improve the oral health of patients. In the present study, it was indicated that the Zufa had equal effectiveness on the oral health of patients under mechanical ventilation as normal saline and Chlorhexidine gluconate. Furthermore, it was reported that normal saline or $0.02 \%$ Zufa extract, which have a lower level of complications compared to Chlorhexidine gluconate, could be used for mild oral health problems of patients under ventilation. However, further research is required to confirm these results.

\subsection{Limitations}

In this study, the remaining oral solution in the mouths was suctioned in less than 30 seconds using a 


\begin{tabular}{|c|c|c|}
\hline Groups/Days & BOAS Mean \pm SD Scores & P Value \\
\hline Zufa & & 0.001 \\
\hline Before the intervention & $6.40 \pm 0.56$ & \\
\hline One day after the intervention & $5.33 \pm 0.47$ & \\
\hline Three days after the intervention & $5.03 \pm 0.18$ & \\
\hline Chlorhexidine & & 0.001 \\
\hline Before the intervention & $6.43 \pm 0.72$ & \\
\hline One day after the intervention & $5.33 \pm 0.54$ & \\
\hline Two days after the intervention & $5.07 \pm 0.25$ & \\
\hline Three days after the intervention & $5.07 \pm 0.25$ & \\
\hline Normal saline & & 0.001 \\
\hline Before the intervention & $6.47 \pm 0.62$ & \\
\hline One day after the intervention & $5.47 \pm 0.62$ & \\
\hline Two days after the intervention & $5.23 \pm 0.43$ & \\
\hline Three days after the intervention & $5.20 \pm 0.40$ & \\
\hline
\end{tabular}

white nelaton catheter. However, during this time, all the solution in the mouth may not have been removed.

\section{Acknowledgments}

We would like to thank the Nursing Care Research Center of Semnan University of Medical Sciences for providing facilities for this work and all patients that participated in this study and their kind cooperation.

\section{Footnotes}

Authors' Contribution: Abbasali Ebrahimian and Niloofar Kianvash-Rad conceived and designed the study, edited the manuscript, supervised the conduct of the study and data collection. Mehrooz Alishah and Alieh Zamani devised and developed the project, and collected the data. Abbasali Ebrahimian wrote the first and final draft of the manuscript. Monir Nobahar undertook recruitment and data collection. Raheb Ghorbani involved in data management and analysis. All authors contributed to reviewing and revising the manuscript, and all took responsibility for the final version.

Conflicts of Interests: The authors declare that they have no competing interests.

Ethical Approval: Before collecting the data, the study protocol was approved by the ethics committee of Semnan University of Medical Sciences (No.:
IR.SEMUMS.REC.1396.5), followed by explaining the objectives of the research to patients and obtaining a written informed consent.

Funding/Support: This study was a part of MSc thesis that was financially supported by a grant (number: 960) from Semnan University of Medical Sciences.

\section{References}

1. Wei HP, Yang K. Effects of different oral care scrubs on ventilatorassociated pneumonia prevention for machinery ventilates patient: A protocol for systematic review, evidence mapping, and network meta-analysis. Medicine (Baltimore). 2019;98(12). e14923. doi: 10.1097/MD.0000000000014923. [PubMed: 30896651].

2. Samaranayake L, Matsubara VH. Normal oral flora and the oral ecosystem. Dent Clin North Am. 2017;61(2):199-215. doi: 10.1016/j.cden.2016.11.002. [PubMed: 28317562].

3. Kouchak F, Askarian M. Nosocomial infections: The definition criteria. Iran J Med Sci. 2012;37(2):72-3. [PubMed: 23115435]. [PubMed Central: PMC3470069].

4. Kelly BJ, Imai I, Bittinger K, Laughlin A, Fuchs BD, Bushman FD, et al. Composition and dynamics of the respiratory tract microbiome in intubated patients. Microbiome. 2016;4:7. doi: 10.1186/s40168-016-0151-8. [PubMed: 26865050]. [PubMed Central: PMC4750361].

5. Aeen FB, Zolfaghari M, Noghabi AA, Mehran A. [Nurses' performance in prevention of ventilator associated pneumonia]. Hayat. 2013;19(3). Persian.

6. Warren C, Medei MK, Wood B, Schutte D. A nurse-driven oral care protocol to reduce hospital-acquired pneumonia. Am J Nurs. 2019;119(2):44-51. doi: 10.1097/01.NAJ.0000553204.21342.01. [PubMed: 30681478].

7. Ronanki S, Kulkarni S, Hemalatha R, Kumar M, Reddy P. Efficacy of commercially available chlorhexidine mouthrinses against specific oral microflora. Indian J Dent Res. 2016;27(1):48-53. doi: 10.4103/09709290.179816. [PubMed: 27054861]. 
8. Chackartchi T, Hamzani Y, Shapira L, Polak D. Effect of subgingival mechanical debridement and local delivery of chlorhexidine gluconate chip or minocycline hydrochloride microspheres in patients enrolled in supportive periodontal therapy: A retrospective analysis. Oral Health Prev Dent. 2019;17(2):167-71. doi: 10.3290/j.ohpd.a42375. [PubMed: 30968072].

9. Chitsazi M, Shirmohammadi A, Balayi E. Effect of herbal and chemical mouth-rinses on periodontal indices; comparison of matrica, persica and chlorhexidine. J Dent Shiraz Univ Med Sci. 2008;8(4):54-60.

10. Kandwal A, Mamgain RK, Mamgain P. Comparative evaluation of turmeric gel with $2 \%$ chlorhexidine gluconate gel for treatment of plaque induced gingivitis: A randomized controlled clinical trial. Ayu. 2015;36(2):145-50. doi: 10.4103/0974-8520.175537. [PubMed: 27011714]. [PubMed Central: PMC4784123].

11. Hua F, Xie H, Worthington HV, Furness S, Zhang Q, Li C. Oral hygiene care for critically ill patients to prevent ventilator-associated pneumonia. Cochrane Database Syst Rev. 2016;10. CD008367. doi: 10.1002/14651858.CD008367.pub3. [PubMed: 27778318]. [PubMed Central: PMC6460950].

12. Deschepper M, Waegeman W, Eeckloo K, Vogelaers D, Blot S. Effects of chlorhexidine gluconate oral care on hospital mortality: A hospitalwide, observational cohort study. Intensive Care Med. 2018;44(7):101726. doi: 10.1007/s00134-018-5171-3. [PubMed: 29744564]. [PubMed Central: PMC6061438].

13. Price R, MacLennan G, Glen J, Su DC. Selective digestive or oropharyngeal decontamination and topical oropharyngeal chlorhexidine for prevention of death in general intensive care: Systematic review and network meta-analysis. BMJ. 2014;348:g2197. doi: 10.1136/bmj.g2197. [PubMed: 24687313]. [PubMed Central: PMC3970764].

14. Bekut M, Brkic S, Kladar N, Dragovic G, Gavaric N, Bozin B. Potential of selected Lamiaceae plants in anti(retro)viral therapy. Pharmacol Res. 2018;133:301-14. doi: 10.1016/j.phrs.2017.12.016. [PubMed: 29258916].

15. Cui Q, Wang LT, Liu JZ, Wang HM, Guo N, Gu CB, et al. Rapid extraction of Amomum tsao-ko essential oil and determination of its chemical composition, antioxidant and antimicrobial activities. J Chromatogr B Analyt Technol Biomed Life Sci. 2017;1061-1062:364-71. doi: 10.1016/j.jchromb.2017.08.001. [PubMed: 28800540].

16. Niazi FH, Kamran MA, Naseem M, AlShahrani I, Fraz TR, Hosein M. Anti-plaque efficacy of herbal mouthwashes compared to synthetic mouthwashes in patients undergoing orthodontic treatment: A randomised controlled trial. Oral Health Prev Dent. 2018;16(5):409-16. doi: 10.3290/j.ohpd.a40983. [PubMed: 30151504].

17. Smida I, Pentelescu C, Pentelescu O, Sweidan A, Oliviero N, Meuric V, et al. Benefits of sea buckthorn (Hippophae rhamnoides) pulp oil-based mouthwash on oral health.JAppl Microbiol. 2019;126(5):1594-605. doi: 10.1111/jam.14210. [PubMed: 30674068].

18. Fathiazad F, Mazandarani M, Hamedeyazdan S. Phytochemical analysis and antioxidant activity of Hyssopus officinalis L. from Iran. Adv Pharm Bull. 2011;1(2):63-7. doi: 10.5681/apb.2011.009. [PubMed: 24312758]. [PubMed Central: PMC3845980].

19. Alinezhad H, Baharfar R, Zare M, Azimi R, Nabavi SF, Nabavi SM. Biochemical activities of acetone extracts of Hyssopus angustifolius. Acta Pol Pharm. 2012;69(4):617-22. [PubMed: 22876603].

20. Lawless J. The Encyclopedia of essential oils: The complete guide to the use of aromatic oils in aromatherapy, herbalism, health, and well being. Conari Press; 2013.

21. Wang N, Yang XW. Two new flavonoid glycosides from the whole herbs of Hyssopus officinalis.J Asian Nat Prod Res. 2010;12(12):1044-50. doi: 10.1080/10286020.2010.533120. [PubMed: 21128145].

22. Kreis W, Kaplan MH, Freeman J, Sun DK, Sarin PS. Inhibition of HIV replication by Hyssop officinalis extracts. Antiviral Res. 1990;14(6):32337. doi:10.1016/0166-3542(90)90051-8. [PubMed:1708226].

23. Vlase L, Benedec D, Hanganu D, Damian G, Csillag I, Sevastre B, et al. Evaluation of antioxidant and antimicrobial activities and phenolic profile for Hyssopus officinalis, Ocimum basilicum and Teucrium chamaedrys. Molecules. 2014;19(5):5490-507. doi: 10.3390/molecules19055490. [PubMed: 24786688]. [PubMed Central: PMC6270679].

24. Safarabadi M, Ghaznavirad E. [Comparing the effect of Echinacea and chlorhexidine mouthwash on oral health in patients hospitalized in intensive care units]. Complement Med J Faculty Nurs Midwifery. 2012;2(3):222-34. Persian.

25. Baradari AG, Khezri HD, Arabi S. Comparison of antibacterial effects of oral rinses chlorhexidine and herbal mouth wash in patients admitted to intensive care unit. Bratisl Lek Listy. 2012;113(9):556-60. doi: 10.4149/BLL_2012_125. [PubMed: 22979913].

26. Vangipuram S, Jha A, Bhashyam M. Comparative efficacy of aloe vera mouthwash and chlorhexidine on periodontal health: A randomized controlled trial. J Clin Exp Dent. 2016;8(4):e442-7. doi: 10.4317/jced.53033. [PubMed: 27703614]. [PubMed Central: PMC5045693]. 